(LETTER TO EDITOR)

\title{
Approach to adult patients admitted with pain to the emergency department
}

\author{
Sogut Ozgur \\ University of Health Sciences, Haseki Training and Research Hospital, Department of Emergency Medicine, Istanbul, \\ Turkey.
}

Publication history: Received on 30 December 2018; revised on 11 February 2019; accepted on 14 February 2019

Article DOI: https://doi.org/10.30574/gscbps.2019.6.2.0165

\begin{abstract}
Pain is the most common complaint among patients presenting to the emergency department (ED). Acute pain activates the sympathetic system and results in increases in blood pressure, heart rate, and respiratory rate, as well as urinary retention, enlarged pupils, and contraction of local muscles. Inadequate management of acute pain may result in thromboembolic or pulmonary complications, prolonged intensive care unit or regular ward stay, hospital readmission for pain following discharge, reduced quality of life, and the development of chronic pain. Effectively provided analgesia is the single intervention that results in patient satisfaction in the ED. In patients with severe pain, opioids can be directly administered intravenously as potent agents; otherwise, per oral-intramuscular non-steroidal antiinflammatory drugs or intravenous-per oral paracetamol may be administered. To reduce analgesia-associated complications in patients encountered in the ED, appropriate analgesics should be titrated and used in appropriate doses.
\end{abstract}

Keywords: Acute pain management; Analgesia; Emergency department; Opioid analgesics; Non-opioid analgesics

\section{Introduction}

Pain is the most common complaint among patients presenting to the emergency department. In a French study, the percentage of patients seen in the emergency department for pain was more than $80 \%$ [1]. According to the definition of the International Association for the Study of Pain, pain is an unpleasant sensory and emotional experience that results from a true or potential tissue injury originating from any site in the body [1,2]. According to the American National Institute of Health, the self-expression of a patient is the most reliable marker of the presence and intensity of pain [3].

Acute pain has a distinct time of onset and is produced by the stimulation of nociceptors, directly or through tissue injury by painful stimuli (e.g., surgery, trauma, acute disease). Acute pain activates the sympathetic system and results in increases in blood pressure, heart rate, and respiratory rate, as well as urinary retention, enlarged pupils, and contraction of local muscles $[4,5]$. Acute pain is not a disorder or syndrome, but a symptom that brings a patient to the physician's attention. It is therefore recognized as the fifth vital sign $[5,6]$.

Inadequate management of acute pain may result in thromboembolic or pulmonary complications, prolonged intensive care unit or regular ward stay, hospital readmission for pain following discharge, reduced quality of life, and the development of chronic pain. Trauma, the most common cause of acute pain, causes sympathetic system activation and thus increases in heart rate, blood pressure, respiratory rate, blood glucose level, and urinary retention. These events lead to worsening clinical status of a trauma patient [6,7]. Similarly, acute pain reduces chest wall and diaphragm motion and may cause atelectasis. Pain also causes tachycardia, increased myocardial oxygen use, hypercoagulability, and increased catabolism in critically ill patients.

\footnotetext{
${ }^{*}$ Corresponding author

E-mail address: ozgur.sogut@sbu.edu.tr
} 
Analgesia reduces perceived pain, but without altering consciousness at any level. However, consciousness may be altered by the secondary effects of drugs used for analgesia [7].

The following steps should therefore be completed before, during, or after analgesia administration [5-7]

- Patient evaluation: history of allergy, medications used, previous disorders, airway abnormalities (short neck, small mandible, large tongue, trismus)

- Preprocedural preparation

- Monitoring: visual and verbal monitoring of consciousness, blood pressure, pulse rate, $\mathrm{SpO2}$, respiratory rate

Conditions requiring analgesia administration in the emergency department are given below $[1,2,4]$

- Acute painful conditions - Renal colic, headache, trauma, acute inflammatory events

- Orthopedic interventions - Fracture, dislocation reduction

- Some wound care procedures - Burn, abscess drainage, foreign body removal

- Endotracheal intubation

- Thoracic tube insertion, central catheter insertion

- Thoracentesis, cardioversion, pericardiocentesis

Analgesic drugs used for acute pain management are divided into three groups

\section{Opioid (narcotic) analgesics}

Drugs in this group have potent central and peripheral effects. Despite their strong analgesic effects, they lack antiinflammatory and anti-pyretic actions. They are used alone or in combination with other analgesics in patients with moderate to severe pain [5-8].

- Morphine

- Meperidine

- Fentanyl

- Tramadol

\section{Non-opioid analgesics}

Acetaminophen (paracetamol)

These widely used non-opioid drugs have proven efficacy in terms of their analgesic and anti-pyretic effects; however, they lack anti-inflammatory effects. These analgesics are used to treat mild to moderate pain $[2,6]$.

Non-steroidal anti-inflammatory drugs (NSAIDs)

\section{Ketamine}

\section{Conclusion}

Acute pain is a condition that should be addressed, after the assessment of airway, breathing and circulation (ABC), as the fourth vital sign. Effectively provided analgesia is the single intervention that results in patient satisfaction in the emergency department. In patients with severe pain, opioids can be directly administered intravenously as potent agents; otherwise, per oral-intramuscular NSAID or intravenous-per oral paracetamol may be administered. To reduce analgesia-associated complications in patients seen in the emergency department, appropriate drugs should be titrated and used in appropriate doses. Patients should be repeatedly evaluated before, during, and after intervention. Altered consciousness, advanced age, and sociocultural status should not preclude analgesia administration.

\section{References}

[1] Milojevic KG, Cantineau JP, Ruiz R, Coudert B, Bataille S, Boutot F, Simon N and Lambert Y. (2004). Can severe acute pain escape visual analog scale screening in the ED? The American Journal of Emergency Medicine, 22(3), 238-241. 
[2] Sogut O, Solduk L, Gokdemir MT and Kaya H. (2015). Impact of single-dose intravenous paracetamol on lymphocyte DNA damage and oxidative stress in trauma patients. Biomedical Research, 26 (1), 23-30.

[3] Cornelius R, Herr KA, Gordon DB, Kretzer K and Butcher HK. (2006). Evidence-Based Practice Guideline: Acute Pain Management in Older Adults. Journal of Gerontological Nursing, 43(2), 18-27.

[4] Berthier F, Potel G, Leconte P, Touze MD and Baron D. (1998). Comparative study of methods of measuring acute pain intensity in an ED. The American Journal of Emergency Medicine; 16(2), 132-136.

[5] Trupkovic T, Kinn M and Kleinschmidt S. (2011). Analgesia and sedation in the intensive care of burn patients: results of a European survey. Journal of Intensive Care Medicine, 26(6), 397-407.

[6] McNicol ED, Ferguson MC, Haroutounian S, Carr DB and Schumann R. (2016). Single dose intravenous paracetamol or intravenous propacetamol for postoperative pain. Cochrane Database of Systematic Reviews, 23(5), CD007126.

[7] Murray MJ. (1990). Pain problems in the ICU. Critical care clinics, 6(2), 235-253.

[8] Pourmand A, Mazer-Amirshahi M, Royall C, Alhawas R and Shesser R. (2017). Low dose ketamine use in the emergency department, a new direction in pain management. The American Journal of Emergency Medicine, 35(6), 918-921.

\section{How to cite this article}

Sogut 0. (2019). Approach to adult patients admitted with pain to the emergency department. GSC Biological and Pharmaceutical Sciences, 6(2), 68-70. 